\title{
La Recherche de la Pureté dans Électre de Jean Giraudoux
}

\author{
Ari M. Abdulrahman ${ }^{1}$ \\ ${ }^{1}$ Department of English language, College of Languages, University of Sulaimani, Kurdistan Region, Iraq
}

\section{RESUME}

Électre, le protagoniste de la pièce de théâtre de Giraudoux, était à la recherche de l'absolu dans son existence. Elle cherchait à rétablir la justice, la pureté et la vérité. Contrairement à tous les autres personnages de la pièce, elle n'a pas accepté le bonheur médiocre qui lui était offert. Sa recherche de la vérité résultait de la recherche de la pureté, en particulier dans sa famille. La plupart des personnages de la pièce souffraient d'impureté dans leurs relations familiales. La famille d'Électre est impure en raison des crimes commis et d'inceste implicite. Elle cherche à retrouver la pureté de la famille, mais la seule solution à cette impureté profondément enracinée est la mort et la destruction totale afin de commencer une nouvelle vie dès le début. La pureté de la pièce est représentée par un certain nombre d'éléments différents tels que la nature, l'enfance et la lumière. L'enfance, qui représente une existence innocente, joue un rôle majeur dans la pièce et constitue la cause principale de conflit entre Électre et sa mère. La nature est le seul élément pur et beau et elle conserve sa pureté tout au long de la pièce, mais elle ne peut toujours fournir un remède au dilemme éprouvé par Électre.

MOTS CLES : Électre, Enfance, Famille, Inceste, Pureté

\section{INTRODUCTION:}

In Jean Giraudoux dans sa pièce Électre s'est concentré principalement sur le refus absolu du bonheur médiocre offert au protagoniste. La plupart des recherches se sont concentrées sur ce thème et ont négligé le thème dominant de la pureté. Dans l'étude actuelle, l'accent sera mis sur ce dernier thème. À cette fin, cette recherche traite principalement de thèmes liés à ce sujet, tels que les crimes au sein de la famille royale, l'inceste, la nature, l'enfance et l'innocence. La recherche commence avec le personnage principal Électre qui cherche désespérément à restaurer la pureté de sa famille. L'analyse commence par la famille royale qui avait été impliquée dans des crimes et des trahisons et l'impact du passé sera expliqué

Koya University Journal of Humanities and Social Sciences (KUJHSS), Volume 4, Issue 1, 2021.

Received 20 Oct 2019; Accepted 31 Aug 2020,

Regular research paper: Published 30 Jun 2021

Corresponding author's e-mail: ariabdulrahman76@gmail.com Copyright $@ 2021$. Ari M. Abdulrahman, this is an open access article distributed under the Creative Commons Attribution License. par rapport à Électre et sa quête de pureté. Ensuite, la recherche traite du thème de l'inceste au sein de la famille royale et comment ce thème peut être un autre élément qui fait de la famille une source de tristesse et de honte pour Électre. La nature est un autre thème essentiel traité dans cette étude. La recherche se concentre sur l'influence et l'effet de la nature sur les personnages principaux de la pièce, en particulier Électre et le jardinier, et comment son influence peut les aider à prendre des décisions. En fin de compte, ce travail traite du thème de l'enfance et de l'innocence et des effets de l'enfance et de l'innocence perdues sur les futurs personnages adultes et comment les souvenirs de cette période déclenchent la révélation de la vérité.

\section{2. ÉLECTRE ET LA RECHERCHE DE LA PURETE}

Jean Giraudoux (1882-1994) a été un romancier et dramaturge français et il a été un dramaturge important durant les deux guerres mondiales. Il s'intéressait principalement à la mythologie grecque et ses thèmes traitent principalement les relations entre l'homme et la femme et des idéaux qui ne pouvaient pas être réalisés. Ce même thème refait surface dans sa pièce Électre (1937), axée sur la recherche de la vérité et de la pureté

Original Article |DOI: $\underline{\text { https://doi.org/10.14500/kujhss.v4n1y2021.pp28-34 }}$ 
par un personnage féminin fort.

Parlant de la raison de la réécriture de la mythologie grecque, Giraudoux dit: «j'ai essayé de rendre la vie à une figure historique que la poussière recouvrait. Admettons que j'ai épousseté le buste d'Électre » (Cité dans Duchêne Hervé, 1997 ; p.64)

Électre, le personnage principal de la pièce est en une quête désespérée de la vérité et de la pureté. Elle tente par tous les moyens de restaurer sa famille et sa pureté. Grandjean (1998, p.25) dit que le nom Électre en langue grecque signifie non-mariée et le fait qu'elle soit encore vierge affirme sa pureté et les femmes vierges au théâtre de Jean Giraudoux sont les symboles de l'innocence et de la liberté.

Le conflit récurrent et très influent entre Électre et sa mère concerne l'accident de la chute d'Oreste. Électre lui rappelle l'incident de laisser Oreste tomber de ses mains sur le marbre « Tu nous portais mal. Tu as laissé tomber Oreste sur le marbre » (Giraudoux, 1987, p.57)

Cela rappelle la recherche de la pureté absolue dans la famille, car Électre accorde trop d'attention à chaque détail de la famille. Elle voudrait que la famille soit totalement pure sans aucune trace d'impureté, mais cela est impossible et la seule solution à sa disposition est de commencer dès le début en l'absence de ceux qui ont été impurs. Et pour attester cette affirmation, Électre parle de la pureté absolue et de l'amour que sa mère devait procurer à sa famille «De toutes mes forces je l'ai retenu. Par sa petite tunique bleue. Par son bras. Par le bout de ses doigts. Par son sillage. Par son ombre. Je sanglotais en le voyant à terre, sa marque rouge au front»> (Giraudoux, p.58). Électre prononce la pureté et la justice absolues en parlant de l'incident de la chute de son frère par sa mère "Si c'est moi qui ai poussé Oreste j'aime mieux mourir, j'aime mieux me tuer... Ma vie n'a aucun sens» (Giraudoux, p.59). Dans cette citation, Électre montre sa conviction de ce qu'une famille doit être. Elle affirme que si elle ne peut pas garder la pureté absolue de la famille, elle préférerait mourir ou se suicider. Ainsi, lorsque la famille pure est rendue impure, la seule solution est la mort pour ceux qui ont contaminé la famille.

Encore une fois le thème de la pureté refait surface quand Électre discute avec sa mère la raison principale de son mariage avec le jardinier « Tu as voulu que je sois dans ton camp. Tu as voulu ne pas avoir perpétuellement devant toi le visage de celle qui est ta pire ennemie...Celui de la chasteté» (Giraudoux, p.86). Ici, Électre se décrit comme le symbole de la pureté pour rappeler à sa mère son impureté. C'est pourquoi sa mère tente d'éliminer cette impureté de sa vie et le seul moyen est de l'épouser au jardinier.

Parlant du personnage d'Électre, Mazon Paul (Cité dans Duchêne Hervé, p.67) précise qu'« elle est gardienne de l'honneur de la famille et se voue dès lors à sa tâche avec une ferveur que rien ne décourage»

Clytemnestre parle des conséquences néfastes de la recherche de la vérité, de la pureté et de la découverte de secrets, tandis qu'Électre parle de l'acceptation des conséquences de la révélation de la vérité «Tout le mal $\mathrm{du}$ monde est venu de ce que les soi-disant purs ont voulu déterrer les secrets et les ont mis en plein soleil » (Giraudoux, p.127)

Clytemnestre affirme à Électre qu'en scandalisant sa mère, elle pollue sa propre image car elle ressemble à sa propre mère et elle confirme que "Le scandale n'est jamais retombé que sur ceux qui le provoquent. À quoi te sert d'éclabousser toutes les femmes en m'éclaboussant ! Tu souilleras pour les yeux d'Oreste tout ce par quoi tu me ressembles »(Giraudoux, p.131). Électre lui répond « je ne te ressemble en rien... et je n'ai pas d'amant » (Giraudoux, p.131). Électre néglige ici la ressemblance physique entre elle et sa mère et affirme qu'elle est différente et ne lui ressemble pas parce qu'elle est pure tandis que sa mère ne l'est pas parce que sa mère $a$ un amant.

La seule raison pour laquelle Électre accepte de se marier avec le jardinier, bien qu'elle connaisse très bien leur intention malveillante, est que le jardinier lui rappelle son père et qu'il est le seul personnage pur dans tout le palais «Je sais que vous avez formé tous deux le projet de me marier au jardinier de mon père. J'accepte » (Giraudoux, p.62). Électre est engourdie; mais pas pour longtemps, en vivant dans les souvenirs de son enfance, de son père et du jardin.

Lorsque les Corinthiens attaquent Argos, Egisthe demande à Électre de le laisser défendre la ville affirmant qu'elle peut se venger plus tard, Électre répond «Compte sur moi. On n'a le droit de sauver une patrie qu'avec des mains pures » (Giraudoux, p.154)

Électre et Egisthe se battent dans deux domaines différents et parlent tous les deux de leurs devoirs. Le devoir d'Égisthe est de sauver son pays et son peuple, tandis qu'Électre a le devoir de révéler la vérité et retrouver la pureté perdue dont Egisthe est la cause.

Egisthe parle de la justice absolue alors qu'Électre tente d'atteindre ou de retrouver la pureté « La justice d'Électre consiste à ressasser toute faute, à rendre tout acte irréparable ? » (Giraudoux, p.170). Ici, Égisthe montre qu'il la connaît très bien et sait ce qu' elle cherche.

Parlant de sa pièce Électre, Giraudoux (Cité dans Sabiani ,2001) dit que «L'humanité, par une faculté d'oubli et par la crainte des complications, résorbe des grands crimes. Mais à chaque époque surgissent des êtres purs qui ne veulent pas que ces grands crimes soient résorbés et empêchent cette résorption... Électre 
est de ces être-là. Elle atteindra son but, mais au prix d'effroyables catastrophes »

Électre et Egisthe vivent dans deux mondes différents. Le monde d'Électre représente l'innocence et la pureté de l'enfance tandis que le monde d'Egisthe est celui de la réalité et de l'âge adulte. Le choc des idées et des croyances de ces deux mondes apparemment différents peut être remarqué dans la discussion entre Électre et Egisthe dans leur définition et leur concept de ce que sont les gens d'une ville.

La quête d'Électre pour une justice et une pureté parfaite se reflète dans ses paroles sur les gens «Quand vous voyez un immense visage emplir l'horizon et vous regarder bien en face, d'yeux intrépides et purs, c'est cela un peuple " (Giraudoux, p.170). Elle ne se soucie pas de la destruction de la ville et même de la mort des gens parce que les gens de sa ville ne sont pas purs. Pour elle, le vrai people est celui qui est pur et qui n'a peur de rien.

Egisthe a une définition et un concept différents de ce que le peuple est « Tu parles en jeune fille, non en roi. $C^{\prime}$ est un immense corps à régir, à nourrir » (Giraudoux, p.170). Egisthe tente à nouveau d'évoquer la pitié et la sympathie d'Électre pour le peuple en l'avertissant du risque de dévoiler des vérités qui pourraient entraîner la perdition du peuple «Il est des vérités qui peuvent tuer un peuple, Électre » (Giraudoux, p.171). Cette scène prouve qu'Egisthe ne connaît pas très bien le caractère d'Électre alors qu'il tente de lui faire avoir pitié des habitants d'Argos et de les sauver de la mort. Mais Électre lui répond en disant que le peuple est déjà mort « Il est des regards de peuple mort qui pour toujours étincellent » (Giraudoux, p.171)

Maillard (1993, p. 91) souligne que « le dialogue entre Électre et Egisthe est la révélation d'un dilemme tragique entre l'absolu et la relative, entre la raison d'Etat et la vengeance personnelle»

La preuve de l'innocence du monde d'Électre réside dans son attitude à l'égard de la calamité que les gens vont subir si on ne permet pas à Egisthe de les secourir. Le refus absolu d'accepter représente un acte puéril quand elle refuse ce que les autres lui disent, quelles que soient les conséquences. Électre a été privée de son père et les autres ne sont donc pas censés être heureux.

Grandjean (p. 20) dit que Jean Giraudoux tire ses sujets de la Grèce antique et presque toutes ses pièces possèdent une vision tragique de l'homme en lutte contre un destin plus puissant que lui et au-delà de ses capacités, mais parfois, l'homme est sans le savoir redevable.

La recherche absolue de la pureté et de la vérité se reflète dans la conversation entre Egisthe et Électre. Il lui demande de lui donner un jour pour sauver la ville et que la vérité puisse être révélée, mais elle n'accepte pas sa demande en disant « Non. C'est aujourd'hui son jour. J'ai déjà trop vu de vérités se flétrir parce qu'elles ont tardé une seconde » (Giraudoux, p.172).

Got (1997, p.30) montre que « alors que l'humanité est faite pour l'oubli et le compromise...Électre va chaque nuit sur la tombe de son père, réveillant ainsi les remords, les aveux, les taches de sang »

Le président, l'un des personnages de la pièce, décrit Électre comme la représentante de la justice, de la générosité et du devoir. Ces trois caractéristiques d'Électre représentent la pureté absolue. Elle essaie de récupérer toutes ces vertus et la seule solution est la destruction de tout ce qui est bon ou mauvais. Tout comme dans un jardin où il existe des fleurs et les mauvaises herbes et la seule solution, selon Électre, consiste à anéantir tout le jardin, des fleurs et les mauvaises herbes comprises, pour commencer un nouveau départ.

\section{UNE FAMILLE CRIMINELLE ET IMPURE}

Électre est une pièce de crimes et d'impureté. Meurtres et trahison sont les traits dominants de la pièce. La protagoniste Électre en est consciente et souffre psychologiquement de ce fait. Elle essaie de retrouver la pureté de sa famille par tous les moyens mais elle ne peut pas changer le passé impur et criminel de sa famille et la seule solution disponible est de éliminer tout; le bien et le mal et le beau et le laid.

\subsection{Le Péché Originel}

L'histoire d'Électre est le dernier acte cruel et brutal d'une série de crimes odieux commis au sein de la famille royale. Au début, Tantale, le roi de Phrygie voulait essayer la sagacité et la justice des dieux et voulait leur donner à manger à son fils Pélops. Les dieux ont découvert le crime et étaient tellement en colère qu'ils lui ont lancé un sortilège de faim tout le temps. Et ils lancent des malheurs sur tous les descendants de la famille Tantale. Puis ils ont ressuscité Pélops.

\subsection{Le Crime d'Atrée}

Pélops a eu deux fils: Thyeste et Atrée. Thyeste séduisit la femme d'Atrée qui s'est vengé en tuant tous les enfants de Thyeste en les cuisinant tous et les servant à la table de leur père qui n'était pas au courant. Seul Egisthe pouvait échapper au massacre. Les dieux ont été horrifiés par cet acte et ils étaient déterminés à punir non seulement Atrée mais toute sa progéniture.

Atrée a eu également deux fils: Agamemnon et Ménélas. Agamemnon a épousé Clytemnestre et Ménélas a épousé Hélène qui s'est enfuie avec son amoureux Paris du fait qui a conduit à la guerre de Troie. Agamemnon a sacrifié sa fille Iphigénie pour 
plaire aux dieux et atteindre Troie. La guerre a duré dix ans et s'est terminée par la victoire des Grecs. Pendant ce temps, Egisthe et Clytemnestre étaient amoureux. Au retour d'Agamemnon de Troie, ils l'ont tué.

Deux points importants se dégagent de ces citations. Le premier point important est la référence à la deuxième fille Iphigénie. Cette fille n'est pas traitée profondément dans cette pièce par rapport au mythe original. Iphigénie était la fille de Clytemnestre et la sœur aînée d'Électre. Elle a été sacrifiée par son père alors qu'il naviguait dans son bateau pour se rendre à Troie parce que la déesse Artémis voulait le punir (comme il avait accidentellement tué un cerf dans un bosquet effrayé de cette déesse) et qu'elle avait interféré dans la direction des vents pour qu'il ne puisse pas se rendre à Troie. Ainsi, pour apaiser la déesse, il devait sacrifier sa fille aînée. Clytemnestre n'a jamais pardonné à son mari d'avoir tué leur fille et elle a été impliquée dans sa mise à mort avec l'aide d'Egisthe, comme un acte de vengeance. Ainsi, nous pouvons remarquer ici l'impureté au sein de la famille royale. Tuant l'innocente Iphigénie était la principale cause de la haine de Clytemnestre envers son mari.

Agathe, une autre femme qui avait éte une victime de son mari le président, représente l'image d'une femme impure en raison d'avoir un amoureux et en même temps des relations avec Egisthe. Elle parle des différences entre elle et son mari «Je suis jolie et il est laid. Je suis jeune et il est vieux. J'ai de l'esprit et il est bête. J'ai une âme et il n'en a pas. Et c'est lui qui a tout. En tout cas il m'a. Et c'est moi qui n'ai rien. En tout cas, je l'ai» (Giraudoux, p.139). Maillard (pp. 81-81) souligne qu'Agathe, en se révoltant contre la médiocrité de la vie et en s'opposant à son mari, ne représente pas seulement elle-même mais toutes les femmes qui cherchent la liberté.

Clytemnestre; avant d'être coupable, elle a été victime de la tyrannie de son mari le roi. Elle a été marginalisée par son mari et privée de sa fille. Ainsi, son complot avec Egisthe pour tuer son mari était la réaction à la souffrance et à la torture subies à cause de son mari.

Parlant des personnages féminins supposés impurs et infidèles à leurs maris, Evrard Franck (1997, p. 45) parlant de Clytemnestre et d'Agathe affirme que «Elles revendiquent toutes deux l'Indépendance, le droit à l'amour, même indigne, immoral et coupable ». Par conséquent, ces deux personnages féminins ont leurs propres raisons d'être infidèles envers leurs maris. Elles ont toutes les deux étés privées de l'humanité et ont été marginalisées. Body (1987, p.137), parlant de la pièce Électre, montre que «on y traite de la condition de la femme dans la société et la place de l'homme dans l'univers »

\section{INCESTE AU SEIN DE LA FAMILLE ROYALE}

Le thème de l'inceste est implicitement traité dans cette pièce dans différentes scènes, en particulier avec le personnage principal Électre, qui ne se rend pas compte qu'elle est exposée à l'impureté en raison de l'amour incestueux que suscite son père.

Tandis qu'Oreste demande à Électre quelle est la raison pour laquelle elle déteste leur mère, Électre répond " $C^{\prime}$ 'est justement ce que je ne peux supporter d'elle, qu'elle m'ait mise au monde. C'est là ma honte...J'aime tout ce qui, dans ma naissance revient à mon père. J'aime comme il s'est dévêtu, de son beau vêtement de noces, comme il s'est couché, comme tout d'un coup pour m'engendrer » (Giraudoux, p.81).

Ici, Électre parle de sa profonde haine pour sa mère et son amour insondable pour son père. Elle emploie des mots suggérant l'inceste; mots qui sont utilisés par une femme en parlant de son mari. Elle a parlé de son père et de ce qu'elle aimait le plus chez lui, comme il se déshabillait, mettait les habits du mariage et dormait.

Le jardinier, pur et naïf, parle d'Électre alors qu' elle se rend tous les soirs au tombeau de son père "Contre quoi s'acharne Électre ? Elle va chaque nuit sur la tombe de son père, et c'est tout ? " (Giraudoux, p.30). Cette citation indique à quel point le jardinier a une pensée pure et superficielle, mais il s'agit en même temps d'une indication implicite de l'inceste puisque Électre se rend à la tombe de son père uniquement pendant la nuit et comme si elle était sa femme.

Egisthe parle du deuil d'Électre à l'égard de son père en la décrivant comme une épouse et non pas comme une fille et Électre ne le nie pas " Crois-tu vraiment qu'il se plaise à te voir le pleurer, non comme une fille, mais comme une épouse ? " (Giraudoux, p.57).

Dans un autre cas, Électre confirme sa haine pour sa mère uniquement et non pour toutes les femmes lorsque son frère lui a de nouveau demandé pourquoi elle détestait autant leur mère "Ce n'est pas que je déteste les femmes, c'est que je déteste ma mère» (Giraudoux, p.81). Électre explique à Oreste que sa mère est la seule femme qu'elle déteste et qu'elle ne déteste pas toutes les autres femmes, et ça parce que sa mère l'a privée de la seule personne qu'elle aimait le plus, c'est son père.

Le père d'Électre était la seule personne qui l'intéressait. Il était à la fois père et amant et, avec sa mort, la vie d'Électre s'achevait. Elle dit à sa mère qu'elle lui a tout volé " $C^{\prime}$ est bien possible, tu $\mathrm{m}^{\prime}$ as tout volé dans la vie» (Giraudoux, p.135).

\section{LA NATURE}

Jean Giraudoux, contrairement aux auteurs grecs, insiste sur l'importance de la nature et crée un personnage (un jardinier) pour exprimer ce fait. La nature fournit aux âmes douloureuses des remèdes, même temporaires. Franck Evrard (p. 85) dit que " 
Giraudoux est sensible à la vie mystérieuse de la nature $\gg$.

La pièce commence avec un étranger (Oreste) qui entre escorté de trois petites filles, au moment où, de l'autre côté, arrivent le jardinier, en costume de fête, et les villageois «Un étranger (Oreste) entre escorté de trois petites filles, au moment où, de l'autre côté, arrivent le jardinier, en costume de fête, et les invités villageois» (Giraudoux, p.13). Si nous examinons les différents éléments mentionnés dans cette citation, nous remarquons que l'enfance représentée par les trois petites filles et le jardinier indique l'importance de la pureté dans le roman, car l'enfance et le jardin représentent la beauté, la vérité et la pureté absolues

Dans la même page, les petites filles décrivent le jardinier comme étant beau "ce qu'il est beau le jardinier » (Giraudoux, p.13). Cela indique l'influence de la nature sur le jardinier qui semble être beau et pur en raison de sa connexion incessante avec le jardin.

L'étranger, qui est Oreste lui-même, parle de la pureté des êtres humains, des animaux et de la nature « On me posait dans un losange de tigres quand j'étais méchant, et dans un hexagone de fleurs quand j'étais sage» (Giraudoux, p.15). Nous remarquons ici le lien entre la pureté des êtres humains représentée par les bons actes et la belle nature en récompense, tandis que les tigres sauvages symbolisent l'impureté ou les mauvaises actions. Ainsi, les enfants apprennent dès leur enfance à se connecter entre eux-mêmes et la nature.

Électre a recours à la nature pour calmer son âme qui souffre. La nature est la seule entité pure et intacte à sa disposition. Elle reste dans une chambre où ses ancêtres ont commis des crimes de meurtre mais la fenêtre de sa chambre donne sur le jardin avec des jasmins «c'est sa fenêtre, la fenêtre aux jasmins... nos, c'est celle de la chambre où Atrée, le premier roi d'Argos, tua les fils de son frère " (Giraudoux, p.16). Nous voyons ici le conflit entre l'impureté de la famille et la pureté de la nature, mais la nature a gagné jusqu'à présent, car elle engourdit Électre avec ses beaux outils tels que les fleurs et leurs odeurs agréables.

Électre, dans sa recherche de la pureté absolue, s'accroche aux souvenirs de famille. Comme nous l'avons mentionné précédemment, la pièce où elle séjourne donne sur la tombe de son père et sur le jardin. Or, cette pièce était autrefois la chambre de son frère, Oreste.

Le jardinier parle de l'effet positif que la nature a sur Électre quand elle est nerveuse "Électre adore mon jardin. Les fleurs, si elle est un peu nerveuse, lui feront du bien» et Agathe lui répond «Mais elle ne fera pas de bien aux fleurs » (Giraudoux, p.33). Ici on constate la naïveté et simplicité du jardinier qui pense que c'est la nature et son jardin qui nourrissent l'âme épuisé d'Électre et l'aident à se calmer. Agathe qui une femme comme Électre sait très bien analyser le caractère d'Électre. Elle sait qu'elle n'est pas là pour réjouir la beauté de la nature mais pour récupérer les mémoires de son père et nourrir son âme de haine et vengeance. Même le président montre cette relation entre Électre et le jardin en disant que les fleurs fournissent à Électre ce dont elle a besoin, à savoir les souvenirs qui nourrissent sa haine "Électre au jardin, c' est la justice et la mémoire entre les fleurs, c'est la haine» (Giraudoux, p.33).

Le jardinier se sent mal quand la mère d'Électre parle mal du jardin et ne le prend que pour quelque chose non vivante. Elle ignore donc la relation étroite qui existe entre l'être humain et la nature. Tandis qu'elle prend le jardin pour quelque chose sans valeur, le jardinier le prend pour quelque chose animée et il personnifie le jardin «Reine, vous pouvez me refuser Électre, mais ce n'est pas loyal de dire du mal d'un jardin qu'on ne connaît pas» (Giraudoux, p.63).

Letoublon (2008, p. 255) indique que le jardinier montre la relation étroite entre l'homme et la nature. Par sa bonté et sa dignité, ce jardinier représente l'humanisme de Giraudoux qui a choisi le plus humble personnage comme porte-parole.

Pour le jardinier, le jardin lui importe plus que sa propre vie et même Électre car il n'a pas peur de défendre son jardin et de le chérir comme si ce jardin est sa propre vie et sa femme qui sont les seules sources de bonheur pour lui «La reine me provoque. Je réponds. Il est ma dot, il est mon honneur, mon jardin ! " (Giraudoux, p.64).

Le jardinier parle à nouveau de l'impact du jardin sur Électre en disant que, tout comme lui, le jardin est devenu l'unique source de bonheur pour Électre mais il ne sait pas que la raison du sourire est que les fleurs la font se souvenir de son père " Je n'ai jamais vu sourire Électre, mais c'est dans mon jardin que j'ai reconnu sur son visage ce qui ressemble le plus au sourire» (Giraudoux, p.65).

Encore une fois, le jardinier compare la nature à l'être humain en mettant l'accent sur la négligence de l'être humain qui est la source de tristesse et de chagrin pour les saisons "Je ne connais guère les êtres, reine, mais je connais les saisons. Il est temps, juste temps dans notre ville de transplanter le malheur » (Giraudoux, p.66). La preuve est qu'avant d'aimer Électre, il vivait en paix avec la beauté de son propre jardin mais après l'avoir connue, il a commencé à souffrir.

En analysant le caractère du jardinier, Debidour (Cité dans Duchêne Hervé, p. 80) affirme que "dans toute la pièce, il n'y a pas de tendresse et de lumière virginale que par le jardinier » et même Samoyault (1997, p.36) affirme que "le jardinier- figure plus biblique que mythologique- affirmerait ainsi que les purs sont en fait les pauvres et les humbles». 
Pour Électre, la pureté de la nature ne peut pas la décourager de chercher à retrouver la pureté perdue de la famille. La nature pourrait fournir un abri temporaire, mais cela ne pourrait pas la faire oublier sa quête de la vérité et de la pureté absolues. Tandis qu'Oreste se montre prêt à accepter la vie et le bonheur médiocre " $\mathrm{Ne}$ plus nous quitter, cela seul compte! Fuyons ce palais. Allons en Thessalie. Tu verras ma maison, perdue dans les roses et les jasmins " (Giraudoux, p.118). Mais Électre rejette le bonheur qui lui est offert et néglige l'importance du bonheur et de la nature «Tu m'as sauvée $\mathrm{du}$ jardinier, Oreste chéri. Ce n'est pas pour me donner aux fleurs » (Giraudoux, p.118).

Maillard (p. 26) parle de la soif d'Électre pour la pureté et affirme qu'il s'agit d'un sentiment de pureté et d'absolu s'opposant au goût ordinaire de la tranquillité et du bonheur.

Dans la conversation entre Électre et sa mère, sa mère regrette qu'Électre n'ait pas de cœur et ne soit mélancolique et elle mentionne le nom de sa deuxième fille, Chrysothémis, qui aimait les fleurs "Par cette fille sans cœur, sans joie! Ah! Heureusement que ma petite Chrysothémis aime les fleurs! » (Giraudoux, p.175).

$\mathrm{Au}$ début, nous faisons référence à la nature par les deux personnages. La mère d'Électre se réfère à la nature, à savoir aux fleurs, car la nature est censée être une source de tranquillité et un recours contre la réalité sombre. C'est pourquoi Électre lui répond qu'elle n'aime pas les fleurs, ce qui implique qu'elle n'est pas prête à accepter la réalité imposée ou l'engourdissement temporaire apporté par la nature

\section{L'ENFANCE ET L'INNOCENCE}

L'enfance est un thème récurrent de cette pièce. Avec la nature, l'enfance est le seul moyen de fuir la réalité sordide imposée à Électre. Le conflit fondamental de la pièce entre Électre et sa mère a pour raison l'enfance et l'innocence. Électre est coincée entre deux mondes; c'est le monde de l'âge adulte et celui de l'enfance. Le monde des adultes la terrifie parce qu'il représente l'impureté et le meurtre; en conséquence, son accrochage au monde de l'enfance qui est pur et loin de la criminalité et de la cruauté des adultes. Par conséquent, les images de cette période dans la vie d'Électre sont encore si vives qu'elles semblent être à l'heure actuelle. Quand sa mère lui dit qu'elle était trop petite pour se souvenir des détails de la chute d'Oreste, elle répondit « Je n'ai pas poussé Oreste! D'au-delà de toute mémoire, je me le rappelle. Ô Oreste, où que tu sois, entends-moi ! Je ne t'ai pas poussé » (Giraudoux, p.58).

La dernière scène entre Électre et les Euménides illustre le problème central ou la préoccupation principale d'Électre. La deuxième Euménide dit à Électre «Te voilà satisfaite, Électre! La ville meurt». Et Électre lui répond « $\mathrm{S}^{\prime} \mathrm{ils}$ sont innocents, ils renaîtront» (Giraudoux, p.190).

Alors que les Euménides reprochent à Électre d'être responsable de la destruction et du massacre dans la ville, Électre assure que ceux qui sont innocents renaîtront. Ici le mot innocent représente la pureté. Selon Électre, tout doit être détruit et brûlé pour pouvoir commencer dès le début. C'est pourquoi elle a choisi cette direction ou cet objectif et n'a accepté aucune concession concernant le sauvetage de la ville.

Encore une fois, les Euménides critiquent Électre pour son arrogance et lui disent qu' elle n'a plus rien et Électre leur répond trois fois « J'ai ma conscience, j' ai Oreste, j' ai la justice, j'ai tout » (Giraudoux, p.191). Mais quand les Euménides lui confirme qu'elle ne va pas se reposer parce qu'elle est coupable et qu'elle ne peut plus voir Oreste elle leur répond «J'ai la justice. J'ai tout » (Giraudoux, p.191).

Cette scène montre que les Euménides n'ont pas très bien compris Électre. En fait, la seule chose qui intéresse Électre est la justice ou la récupération de la pureté à travers la destruction de tout. Quand elle a mentionné le mot conscience, elle faisait référence à la satisfaction qu'elle tirait de la vengeance et elle ne se souciait jamais de ceux qui avaient été tués car pour elle, ils n'étaient pas purs et méritaient de mourir. En ce qui concerne son frère Oreste, il est vrai qu'elle l'aime mais elle se soucie surtout d'elle-même, car elle veut rester pure et innocente en faisant son frère commettre le crime de de tuer leur mère. Got (p. 47) montre qu'Oreste est un faible, sans passé, sans personnalité; elle peut le faire à sa guise. Ainsi, à la fin, elle ne meurt ni ne se suicide, car, selon elle, elle est toujours pure et innocente, contrairement à ceux qui viennent d'être tués.

Le dernier mot de la pièce est le mot l'aube. La pièce a commencé avec l'innocence et s'est terminé avec le mot aube qui tous les deux représentent la pureté. Le mot aube ici nous rappelle le mot renaissance utilisé par Électre. Maillard (p. 93) montre qu'Électre n'a jamais commis de crime et qu'elle a été innocente affirmant que sa pureté était absolue.

\section{ONCLUSION}

La recherche de la restauration de la pureté était la préoccupation ultime du personnage principal de la pièce, mais il y a eu de nombreux obstacles devant Électre dans ses tentatives pour atteindre ses objectifs. La pureté était représentée par un certain nombre d'éléments tels que l'histoire familiale, l'inceste, la nature, l'enfance et l'innocence. La principale préoccupation d'Électre était l'intégrité de sa famille et en raison de l'histoire sanglante de sa famille, elle ne pouvait pas restaurer cette intégrité et ses efforts se sont soldés par le meurtre de sa mère et la destruction de la ville. L'inceste, bien qu'implicitement traité dans cette 
pièce, ajoute à l'impureté de la famille. Le dilemme d'Électre est qu'elle a aimé son père comme s'il était son mari et qu'elle a détesté sa mère pour lui avoir enlevé son amour deux fois; une fois qu'il était vivant et quand elle l'a tué. La nature est l'un des thèmes essentiels de la pièce et elle est fortement liée à la recherche de la pureté. La nature garde sa pureté tout au long de la pièce et elle fournit un abri aux âmes souffrantes en particulier au personnage principal Électre. La nature lui rappelle son père et elle garde sa mémoire vivante en elle. La pureté et la signification de la nature se voient principalement avec le jardinier qui chérit son jardin, le personnifie et souffre à cause de la négligence et des malentendus des gens à ce sujet. Pourtant, la nature ne peut pas remplacer la recherche de la pureté ultime par Électre car elle n'accepte pas le bonheur offert par la nature et cherche la destruction de tout ce qui est beau afin de venger la mort de son père. L'enfance joue un rôle important dans cette pièce car elle permet de révéler la vérité recherchée par Électre qui est coincée entre deux mondes; le monde des adultes et le monde de l'enfance. Elle s'accroche à l'enfance car c'est le monde de la pureté et rejette le monde des adultes qui est un monde de trahison, de crimes et d'impureté. L'entêtement d'Électre est celui d'un enfant qui dit non et ne cède pas aux désirs des autres. En fin de compte, la persistance d'Électre conduit à la destruction de tout, y compris sa famille.

\section{REFERENCES}

Body Jacques, (1987). Jean Giraudoux, Électre. Imprimé en France, Grasset

Duchêne Hervé, (1997). Électre, Jean Giraudoux. Imprimé en France, BREAL Rosny,

Evrard Franck, (1997). Électre de Sophocle à Giraudoux, Imprimé en C.E.E, Bertrand-Lacoste

Got Olivier, (1997). Etude sur Jean Giraudoux, Électre. Imprimé en France, ellipses

Grandjean Philippe, (1998). Électre, Giraudoux. Profil d'une œuvre, Saint-Amand, France: Bussiere Camedan Imprimeries

Letoublon Françoise, (2008). Électre, Jean Giraudoux. Paris, France: Larousse

Maillard Michel, (1993).Électre, Jean Giraudoux. Imprimé en France, Nathan

Sabiani Marie-Anne, (2001). Sophocle, Électre. Imprimé en France, Breal

Samoyault Tiphaine, (1997). Électre de Giraudoux. Imprimé en France: Hachette

Watt, I., (1957). “The Rise of The Novel”. London: Random House. 